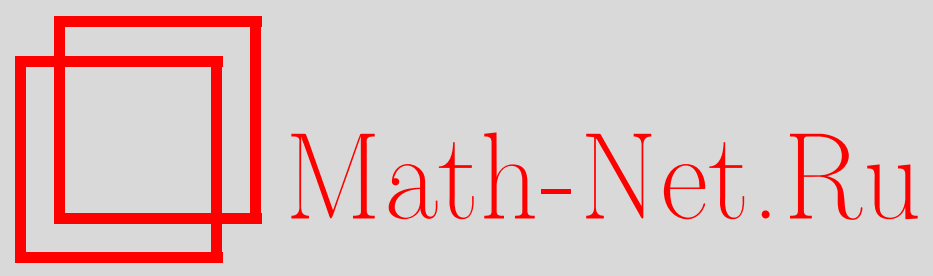

А. Г. Баскаков, Об обратимости и фредгольмовости разностных операторов, Матем. заметки, 2000, том 67, выпуск $6,816-827$

DOI: https://doi.org/10.4213/mzm900

Использование Общероссийского математического портала Math-Net.Ru подразумевает, что вы прочитали и согласны с пользовательским соглашением http://www.mathnet.ru/rus/agreement

Параметры загрузки:

IP : 107.22 .136 .117

26 апреля 2023 г., $13: 14: 37$

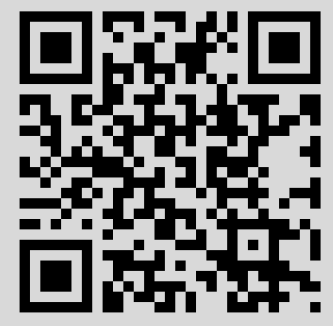




\section{ОБ ОБРАТИМОСТИ И ФРЕДГОЛЬМОВОСТИ РАЗНОСТНЫХ ОПЕРАТОРОВ}

\section{А. Г. Баскаков}

В статье получены условия обратимости и фредгольмовости разностного оператора $(\mathscr{D} x)(n)=x(n)-U(n) x(n-1), n \in \mathbb{Z}$, действующего в банаховом пространстве векторных последовательностей $\ell_{p}(\mathbb{Z}, X), p \in[1, \infty]$, где $X$ - банахово пространство и $U$-ограниченная операторнозначная функция.

Библиография: 8 названий.

Пусть $X$ - комплексное банахово пространство и End $X$ - банахова алгебра эндоморфизмов (линейных ограниченных операторов), действующих в $X$. Символом $\mathcal{N}$ обозначим одно из множеств: $\mathbb{Z}$ - множество целых чисел, $\mathbb{Z}_{+}=\{n \in \mathbb{Z} \mid n \geqslant 0\}=\mathbb{N} \cup\{0\}$, $\mathbb{Z}_{-}=\mathbb{Z} \backslash \mathbb{Z}_{+}=\{n \in \mathbb{Z} \mid n<0\}$.

Через $\mathscr{F}=\mathscr{F}(\mathscr{N}, X)$ обозначим одно из банаховых пространств последовательностей векторов из $X$ следующего вида:

$$
\begin{aligned}
\ell_{p} & =\ell_{p}(\mathcal{N}, X)=\left\{x: \mathscr{N} \rightarrow X \mid\|x\|_{p}=\left(\sum_{n \in \mathcal{N}}\|x(n)\|^{p}\right)^{1 / p}<\infty\right\}, \quad p \in[1, \infty), \\
\ell_{\infty}=\ell_{\infty}(\mathcal{N}, X) & =\left\{x: \mathscr{N} \rightarrow X \mid\|x\|_{\infty}=\sup _{n \in \mathcal{N}}\|x(n)\|<\infty\right\}, \\
c_{0} & =c_{0}(\mathscr{N}, X)=\left\{x \in \ell_{\infty} \mid \lim _{|n| \rightarrow \infty}\|x(n)\|=0\right\} .
\end{aligned}
$$

В данной статье получены условия обратимости и фредгольмовости разностного оператора $\mathscr{D} \in$ End $\mathscr{F}(\mathbb{Z}, X)$, определенного формулой

$$
(\mathscr{D} x)(n)=x(n)-U(n) x(n-1), \quad x \in \mathscr{F}(\mathbb{Z}, X), \quad n \in \mathbb{Z},
$$

где $U: \mathbb{Z} \rightarrow \operatorname{End} X-$ ограниченная функция.

Важная роль при исследовании оператора $\mathscr{D}$ отводится семейству эволюционных операторов $\mathscr{U}=\{\mathscr{U}(n, m),-\infty<m \leqslant n<\infty\}$ из алгебры End $X$, которое определяется с помощью равенств

$$
\mathscr{U}(n, m)= \begin{cases}U(n) U(n-1) \cdots U(m+1), & m<n, \\ I, & m=n,\end{cases}
$$


где $I$ - тождественньй оператор в $X$ (символ $I$ используется для обозначения тождественного оператора в любом из рассматриваемых банаховых пространств).

Непосредственно из определения семейства эволюционных операторов $\mathscr{U}$ следует, что ядро $\operatorname{Ker} \mathscr{D}$ оператора $\mathscr{D}$ допускает представление вида

$$
\operatorname{Ker} \mathscr{D}=\{x \in \mathscr{F}(\mathbb{Z}, X) \mid x(n)=\mathscr{U}(n, m) x(m) \forall m \leqslant n\}
$$

Исследование обратимости и фредгольмовости оператора $\mathscr{D}$ будет осуществляться, используя

ОПРЕДЕЛЕНИЕ 1. Будем говорить, что семейство эволюционных операторов $\mathscr{U} \partial о-$ пускает әкспоненииальную дихотомию на множестве $\mathscr{N}_{0}$ из $\mathbb{Z}$ с постоянньми $M>0$, $q \in(0,1)$, если существует ограниченная проекторнозначная функция $P: \mathscr{N}_{0} \rightarrow$ End $X$ со свойствами:

1) $\mathscr{U}(n, m) P(m)=P(n) \mathscr{U}(n, m)$ для всех $m \leqslant n$ из $\mathscr{N}_{0}$;

2) $\|\mathscr{U}(n, m) P(m)\| \leqslant M q^{n-m}, m \leqslant n, m, n \in \mathscr{N}_{0}$;

3) при $n>m$ из $\mathscr{N}_{0}$ сужение $\mathscr{U}_{n, m}: X^{\prime}(m) \rightarrow X^{\prime}(n)$ оператора $\mathscr{U}(n, m)$ на образ $X^{\prime}(m)=\operatorname{Im} Q(m)$ дополнительного проектора $Q(m)=I-P(m)$ к проектору $P(m)$ является изоморфизмом подпространств $X^{\prime}(m)$ и $X^{\prime}(n)$; определим оператор $\mathcal{U}(m, n) \in$ End $X$ как оператор, совпадающий с $\mathscr{U}_{n, m}^{-1}$ на $X^{\prime}(n)$ и являющийся нулевым на подпространстве $X(n)=\operatorname{Im} P(n)$;

4) $\|\mathscr{U}(m, n)\| \leqslant M q^{n-m}, n>m, m, n \in \mathcal{N}_{0}$.

Пару проекторнозначных функций $(P, Q)$, участвуюших в определении 1 , назовем расщ,епляющей парой для семейства $\mathscr{U}$. Если $P=0$ или $Q=0$, то будем говорить, что для $\mathscr{U}$ имеет место тривиальная дихотомия на множестве $\mathscr{N}_{0}$.

В монографии [1, теорема 7.6.5] приведен следующий результат.

Лемма 1. Onератор $\mathscr{D} \in \operatorname{End} \ell_{\infty}(\mathbb{Z}, X)$ обратим тогда и только тогда, когда семейство $U$ допускает әкспоненциальную дихотомию на множсестве $\mathbb{Z}$.

Проверка свойства экспоненциальной дихотомии семейства $\mathscr{U}$ на $\mathbb{Z}$ весьма затруднительна. Значительно проще проверка вьполнения одного из следующих предположений.

ПРЕДПОЛОЖЕНИЕ 1. Семейство $\mathscr{U}$ допускает әкспоненииальную дихотомию на множествах $\mathbb{Z}_{-} u \mathbb{Z}_{+}$с соответствующими парами расщепляющих функиий $\left(P_{-}, Q_{-}\right),\left(P_{+}, Q_{+}\right)$.

ПРЕДПОЛОЖЕНИЕ 2. Семейство $\mathscr{U}$ допускает әкспоненииальную дихотомию на множествах $\mathbb{Z}_{-} \cup\{0\} \quad и \mathbb{Z}_{+}$с соответствующими парами расщепляющих функuий $\left(P_{-}, Q_{-}\right),\left(P_{+}, Q_{+}\right)$.

Далее используются обозначения

$$
\begin{array}{ll}
X_{-}(n)=\operatorname{Im} P_{-}(n), \quad n \leqslant 0, & X_{+}(n)=\operatorname{Im} P_{+}(n), \quad n \geqslant 0, \\
X_{-}^{\prime}(n)=\operatorname{Im} Q_{-}(n), \quad n \leqslant 0, & X_{+}^{\prime}(n)=\operatorname{Im} Q_{+}(n), \quad n \geqslant 0 .
\end{array}
$$

Следующие три теоремы являются одними из основных результатов данной статьи. 
TEорема 1. Onepamop $\mathscr{D} \in$ End $\mathscr{F}(\mathbb{Z}, X)$ обратим тогда и только тогда, когда выполнено предположсене 1 и линейный оператор

$$
\Phi=Q_{+}(0) U(0) Q_{-}(-1): X_{-}^{\prime}(-1) \rightarrow X_{+}^{\prime}(0)
$$

является изоморфизмом.

Отметим, что обратимость оператора $\mathscr{D}$ означает, что его ядро $\operatorname{Ker} \mathscr{D}=\{0\}$ и его образ $\operatorname{Im} \mathscr{D}$ совпадает с $\mathscr{F}(\mathbb{Z}, X)$. Следовательно, существует ограниченный обратный $\mathscr{D}^{-1} \in$ End $\mathscr{F}$.

Оператор $\Phi$, определенньй формулой (1), назовем узловым оператором.

СлЕдСТвИЕ 1. Onератор $\mathscr{D} \in$ End $\mathscr{F}(\mathbb{Z}, X)$ обратим, если выполнено предположение 1 и $X=U(0) X_{-}^{\prime}(1) \oplus X_{+}(0)$.

СледСТВИЕ 2. Onератор $\mathscr{D} \in$ End $\mathscr{F}(\mathbb{Z}, X)$ обратим, если выполнено предположсние 2 и одно из следующих условий:

1) $X=X_{-}^{\prime}(0) \oplus X_{+}(0)$;

2) onepamop $Q_{-}(0)-P_{+}(0) \in$ End $X$ обратим.

ТеОрема 2. Oператор D обратим при выполнении одного из следующих условиนั:

1) выполнено предполохение 1 и $Q_{-}=0, Q_{+}=0$;

2) выполнено предположсение 2 и $P_{-}=0, P_{+}=0$.

TEOPEмA 3. Onератор $\mathscr{D} \in$ End $\mathscr{F}(\mathbb{Z}, X)$ обратим, если существуют предель

$$
\lim _{n \rightarrow \pm \infty} U(n)=U_{ \pm}
$$

в равномерной операторной топологии и спектральные радиусы $r\left(U_{ \pm}\right)$предельных операторов $U_{ \pm}$удовлетворяют условию $r\left(U_{ \pm}\right)<1$.

Будем говорить, что для решений однородного уравнения

$$
x(n)=U(n) x(n-1), \quad n \geqslant n_{0} \in \mathbb{Z},
$$

имеет место равномерная әкспоненииальная устойчивость, если существуют постоянные $M_{0}>0, q_{0} \in(0,1)$ такие, что для любого решения $x_{0}$ уравнения $(3)$ верна оценка

$$
\left\|x_{0}(n)\right\| \leqslant M_{0} q_{0}^{n-m}\left\|x_{0}(m)\right\| \quad \forall n \geqslant m \geqslant n_{0} .
$$

СлЕДСТВИЕ 3. Если выполнено условие 1) теоремы 2 или условия теоремы 3, то решения уравнения (3) равномерно әкспоненииально устойчивы.

1. О ядре оператора $\mathscr{D}$. Для изучения оператора $\mathscr{D} \in \operatorname{End} \mathscr{F}(\mathbb{Z}, X)$ введем в рассмотрение два разностных оператора $\mathscr{D}_{ \pm} \in \operatorname{End} \mathscr{F}\left(\mathbb{Z}_{ \pm}, X\right)$, которые определяются равенствами

$$
\begin{gathered}
\left(\mathscr{D}_{-} x\right)(n)=x(n)-U(n) x(n-1), \quad n \leqslant-1, \quad x \in \mathscr{F}\left(\mathbb{Z}_{-}, X\right), \\
\left(\mathscr{D}_{+} x\right)(n)= \begin{cases}x(0), & n=0, \\
x(n)-U(n) x(n-1), & n \geqslant 1, x \in \mathscr{F}\left(\mathbb{Z}_{+}, X\right) .\end{cases}
\end{gathered}
$$


ЛЕмма 2. Если семейство И допускает әкспоненциальную дихотомию на множсестве $\mathbb{Z}_{-}$, то оператор $\mathscr{D}_{-}$сюргективен $\left(\right.$т.е. $\left.\operatorname{Im} \mathscr{D}_{-}=\mathscr{F}\left(\mathbb{Z}{ }_{-}, X\right)\right)$, обратим справа и один из правых обратных $\mathscr{B}_{r} \in \operatorname{End} \mathscr{F}\left(\mathbb{Z}_{-}, X\right)$ имеет вид

$$
\left(\mathscr{B}_{r} y\right)(n)=\sum_{m=-\infty}^{-1} G_{r}(n, m) y(m), \quad n \in \mathbb{Z}_{-}, \quad y \in \mathscr{F}\left(\mathbb{Z}_{-}, X\right) .
$$

Oператор $\mathscr{P}_{r}=I-\mathscr{B}_{r} \mathscr{D}_{-}$является проектором, $\operatorname{Im} \mathscr{P}_{r}=\operatorname{Ker} \mathscr{D}_{-}$, и он определяется формулой

$$
\left(\mathscr{P}_{r} x\right)(n)=\mathscr{U}(n,-1) Q_{-}(-1) x(-1), \quad n \leqslant-1, \quad x \in \mathscr{F}\left(\mathbb{Z}_{-}, X\right) .
$$

В условиях леммы функция $G_{r}: \mathbb{Z}_{-} \times \mathbb{Z}_{-} \rightarrow$ End $X$ определяется равенствами

$$
G_{r}(n, m)= \begin{cases}\mathscr{U}(n, m) P_{-}(m), & -\infty<m \leqslant n \leqslant-1, \\ -\mathscr{U}(n, m) Q_{-}(m), & -\infty<n<m \leqslant-1,\end{cases}
$$

где $P_{-}, Q_{-}: \mathbb{Z}_{-} \rightarrow$ End $X-$ расщепляющая пара проекторнозначных функций.

СлЕдСТВИЕ 4. Ядро Ker $\mathscr{D}_{-}$оператора $\mathscr{D}_{-}$состоит из последовательностей $x_{0}(n)=\mathscr{U}(n,-1) Q_{-}(-1) x_{0}, n \leqslant-1, x_{0} \in X$, а любое решение $x$ уравнения

$$
\mathscr{D}_{-} x=y \in \mathscr{F}\left(\mathbb{Z}_{-}, X\right)
$$

имеет вид $x=\mathscr{B}_{r} y+x_{0}, x_{0} \in \operatorname{Ker} \mathscr{D}$.

ДокАЗАТЕЛЬСТВО ЛЕМмы 2 осуществляется непосредственной проверкой того, что оператор $\mathscr{B}_{r}$ является правым обратньм для оператора $\mathscr{D}_{-}$и последующим вычислением формулы для проектора $\mathscr{P}$ - (подробности см. в [2]). Такое же замечание верно и для следующей леммы.

Линейньй оператор $A \in$ End $Y$, действующий в банаховом пространстве $Y$, назовем равномерно инбективным, если существует постоянная $c>0$ такая, что $\|x\| \leqslant c\|A x\|$ $\forall x \in Y$.

Отметим, что в статье [3] для равномерно инъективных дифференциальных операторов В.В. Жиковым использовался термин “корректный.

Лемма 3. Если семейство И допускает әкспоненииальную дихотомию на множестве $\mathbb{Z}_{+}$, то оператор $\mathscr{D}_{+} \in$ End $\mathscr{F}\left(\mathbb{Z}_{+}, X\right)$ равномерно инбективен, обратим слева и один из левых обратных $\mathscr{B}_{l} \in$ End $\mathscr{F}\left(\mathbb{Z}_{+}, X\right)$ представим формулой

$$
\left(\mathscr{B}_{l} y\right)(n)=\sum_{m=0}^{\infty} G_{l}(n, m) y(m), \quad y \in \mathscr{F}\left(\mathbb{Z}_{+}, X\right), \quad n \geqslant 0,
$$

әде функиия $G_{l}$ имеет вид

$$
G_{l}(n, m)= \begin{cases}\mathscr{U}(n, m) P_{+}(m), & 0 \leqslant m \leqslant n<\infty \\ -\mathscr{U}(n, m) Q_{+}(m), & 0 \leqslant n<m<\infty\end{cases}
$$

u $P_{+}, Q_{+}: \mathbb{Z}_{+} \rightarrow$ End $X$ - расщепляющая пара проекторнозначных функиий. Оператор $\mathscr{P}_{l}=\mathscr{D}_{+} \mathscr{B}_{l}-$ проектор на образ $\operatorname{Im} \mathscr{D}_{+}$оператора $\mathscr{D}_{+}$, и он допускает представление вида

$$
\left(\mathscr{P}_{l} x\right)(n)= \begin{cases}x(n), & n \geqslant 1, \\ x(0)-\sum_{m=0}^{\infty} \mathscr{U}(0, m) Q_{+}(m) x(m), & n=0 .\end{cases}
$$


СлЕДСТВИЕ 5. Подпространство $\operatorname{Im} \mathscr{D}_{+}$состоит из последовательностей вида

$$
\left\{\left(x(0)-\sum_{m=0}^{\infty} \mathscr{U}(0, m) Q_{+}(m) x(m), x(1), x(2), \ldots\right), x \in \mathscr{F}\left(\mathbb{Z}_{+}, X\right)\right\}
$$

ЗАмечАниЕ 1. Банахово пространство $\mathscr{F}(\mathbb{Z}, X)$ представимо (изометрически изоморфно) в виде декартова произведения $\mathscr{F}_{-} \times \mathscr{F}_{+}$банаховых пространств $\mathscr{F}_{ \pm}=$ $\mathscr{F}\left(\mathbb{Z}_{ \pm}, X\right)$. Относительно такого представления оператор $\mathscr{D}$ может быть представлен матрицей вида

$$
\left(\begin{array}{cc}
\mathscr{D}_{-} & 0 \\
\mathscr{D}_{0} & \mathscr{D}_{+}
\end{array}\right)
$$

где $\mathscr{D}_{ \pm} \in$ End $\mathscr{F} \pm$ определены формулами $(4),(5)$ и линейный ограниченньй оператор $\mathscr{D}_{0}: \mathscr{F}_{-} \rightarrow \mathscr{F}_{+}$имеет вид

$$
\left(\mathscr{D}_{0} x\right)(n)= \begin{cases}-U(0) x(-1), & n=0 \\ 0, & n \geqslant 1\end{cases}
$$

Матрица (6) оператора $\mathscr{D}$ будет использоваться постоянно, и далее будут рассматриваться матрицы ограниченных операторов из End $\mathscr{F}$, которые определяются представлением $\mathscr{F}=\mathscr{F}_{-} \times \mathscr{F}_{+}$.

ТЕОрема 4. Пусть выполнено предположение 1. Тогда ядро Ker $\mathscr{D}$ оператора $\mathscr{D}$ состоит из последовательностей $x \in \mathscr{F}(\mathbb{Z}, X)$ вида

$$
x(n)= \begin{cases}\mathscr{U}(n,-1) x_{0}, & n \leqslant-1, \\ \mathscr{U}(n, 0) U(0) x_{0}, & n \geqslant 0\end{cases}
$$

әде $x_{0}-$ произвольный вектор из ядра $\operatorname{Ker} \Phi$ оператора

$$
\Phi=Q_{+}(0) U(0) Q_{-}(-1): X_{-}^{\prime}(-1) \rightarrow X_{+}^{\prime}(0)
$$

ДокАЗАТЕЛЬСтво. Рассмотрим оператор $\mathscr{B} \in$ End $\mathscr{F}(\mathbb{Z}, X)$, имеющий матрицу вида

$$
\left(\begin{array}{cc}
\mathscr{B}_{r} & 0 \\
-\mathscr{B}_{l} \mathscr{D}_{0} \mathscr{B}_{r} & \mathscr{B}_{l}
\end{array}\right)
$$

где операторы $\mathscr{B}_{r}$ и $\mathscr{B}_{l}$ были введены в леммах 2 и 3 . Линейньй оператор $\mathscr{A}=I-\mathscr{B} \mathscr{D} \in$ End $\mathscr{F}(\mathbb{Z}, X)$ представим матрицей

$$
\left(\begin{array}{cc}
\mathscr{P}_{r} & 0 \\
-\mathscr{B}_{l} \mathscr{D}_{0} \mathscr{P}_{r} & 0
\end{array}\right)
$$

где проектор $\mathscr{P}_{r} \in$ End $\mathscr{F}_{-}$задан в лемме 2 . Поскольку оператор $\mathscr{B}_{l} \mathscr{D}_{0} \mathscr{P}_{r}: \mathscr{F}_{-} \rightarrow \mathscr{F}_{+}$ определяется формулой

$$
\left(\mathscr{B}_{l} \mathscr{D}_{0} \mathscr{P}_{r} x\right)(n)=\mathscr{U}(n, 0) P_{+}(0) U(0) Q_{-}(-1) x(-1), \quad n \geqslant 0
$$


то оператор $\mathscr{A}$ имеет вид

$$
(\mathscr{A} x)(n)= \begin{cases}\mathscr{U}(n,-1) Q_{-}(-1) x(-1), & n \leqslant-1 \\ \mathcal{U}(n, 0) P_{+}(0) U(0) Q_{-}(-1) x(-1), & n \geqslant 0 .\end{cases}
$$

Из определения этого оператора следует, что $\operatorname{Ker} \mathscr{D} \subset \operatorname{Im} \mathscr{A}$. Таким образом, для описания подпространства $\operatorname{Ker} \mathscr{D}$ достаточно найти условия, при которых последовательности вида $(\mathscr{D} \mathscr{A} y), y \in \mathscr{F}(\mathbb{Z}, X)$, нулевые.

Непосредственная проверка показьвает, что верны равенства

$$
(\mathscr{D} \mathscr{A} y)(n)=0, \quad y \in \mathscr{F}(\mathbb{Z}, X), \quad 0 \neq n \in \mathbb{Z}
$$

При $n=0$ получаем, что условие $(\mathscr{D A} y)(0)=0$ эквивалентно условию (учитьвается, что $\left.P_{+}(0)+Q_{+}(0)=I\right)$

$$
\Phi Q_{-}(-1) y(-1)=Q_{+}(0) U(0) Q_{-}(-1) y(-1)=0 .
$$

Таким образом, каждая последовательность $x \in \operatorname{Ker} \mathscr{D}$ имеет вид (7). Из предположения 1 , влекущего экспоненциальную дихотомию на $\mathbb{Z}_{-}$и $\mathbb{Z}_{+}$, вытекает, что существуют постоянные $M_{0}>0, q_{0} \in(0,1)$ такие, что $\left\|x_{0}(n)\right\| \leqslant M_{0} q_{0}^{|n|}, n \in \mathbb{Z}$. Следовательно, $x_{0} \in \mathscr{F}(\mathbb{Z}, X)$ при любом рассматриваемом нами пространстве $\mathscr{F}(\mathbb{Z}, X)$. Теорема доказана.

ЗАмЕчАнИЕ 2. Введем в рассмотрениеподпространство $X_{0}=U(0) X_{-}^{\prime}(-1) \cap X_{+}(0)$. Если вьполнено предположение 2 , то $U(0) X_{-}^{\prime}(-1)=X_{-}^{\prime}(0)$ и тогда $X_{0}=X_{-}^{\prime}(0) \cap X_{+}(0)$. Непосредственно из определения узлового оператора $\Phi$ следует, что $\operatorname{Ker} \Phi=X_{0}$.

Теперь приведем несколько следствий из теоремы 4.

СлЕДСтвИЕ 6. Имеют место равенства $\operatorname{dim} \operatorname{Ker} \mathscr{D}=\operatorname{dim} \operatorname{Ker} \Phi=\operatorname{dim} X_{0}$.

СлЕДСТВИЕ 7. Если выполнено предположение 2, то

$$
\operatorname{dim} \operatorname{Ker} \mathscr{D}=\operatorname{dim}\left(X_{-}^{\prime}(0) \cap X_{+}(0)\right)
$$

СлЕДСТВИЕ 8. Если размерность подпространства $U(0) X_{-}^{\prime}(-1)$ конечна, то $\operatorname{dim} \operatorname{Ker} \mathscr{D}<\infty$.

СлЕДСТВИЕ 9. Если оператор $U(0)$ компактен, mо $\operatorname{dim} \operatorname{Ker} \mathscr{D}<\infty$.

СЛЕДСтвИЕ 10. Если $x_{0} \in \operatorname{Ker} \mathscr{D}$, то существуют постоянные $M_{0}>0, q_{0} \in(0,1)$ maкие, что $\left\|x_{0}(n)\right\| \leqslant M_{0} q_{0}^{|n|}, n \in \mathbb{Z}$.

СлЕдСТвИЕ 11. Ядро Ker $\mathscr{D}$ оператора $\mathscr{D}$ не зависит от выбора пространства $\mathscr{F}(\mathbb{Z}, X)$.

СледСтвие 12. Onepamop $\mathscr{A}=I-\mathscr{B} \mathscr{D} \in$ End $\mathscr{F}(\mathbb{Z}, X)(\mathscr{A}, \mathscr{B}$ введены при доказательстве теоремы 3) конечномерен (следовательно, компактен), если

$$
\operatorname{dim} X_{-}^{\prime}(-1)<\infty .
$$


ТЕорема 5. Пусть выполнено предположение 1. Оператор Ф равномерно инбективен тогда и только тогда, когда равномерно инбективен узловой операmop $\Phi$.

ДокАЗАТЕЛьСтво. Вернемся к доказательству теоремы 4 и используем введенные там операторы.

Пусть вначале равномерно инъективен оператор $\Phi$. Предположим, что $\mathscr{D}$ не является равномерно инъективным, т.е. существует нормированная последовательность $\left(x_{n}\right)$ из $\mathscr{F}(\mathbb{Z}, X)$ такая, что

$$
\lim _{n \rightarrow \infty}\left\|\mathscr{D} x_{n}\right\|=0
$$

Тогда

$$
\lim _{n \rightarrow \infty}\left\|\mathscr{A} x_{n}-x_{n}\right\|=0
$$

и поэтому

$$
\lim _{n \rightarrow \infty}\left\|\mathscr{D} \mathscr{A} x_{n}\right\|=0
$$

В частности, из равенств

$$
\begin{aligned}
(\mathscr{D} \mathscr{A} x)(0) & =(\mathscr{A} x)(0)-U(0)(\mathscr{A} x)(-1)=P_{+}(0) U(0) Q_{-}(-1) x(-1)-U(0) Q_{-}(-1) x(-1) \\
& =-Q_{+}(0) U(0) Q_{-}(-1) x(-1)=-\Phi Q_{-}(-1) x(-1)
\end{aligned}
$$

получаем, что

$$
\lim _{n \rightarrow \infty}\left\|\mathscr{D} \mathscr{A} x_{n}(0)\right\|=\lim _{n \rightarrow \infty}\left\|\Phi Q_{-}(-1) x_{n}(-1)\right\|=0 .
$$

Из равномерной инъективности оператора $\Phi$ следует, что

$$
\lim _{n \rightarrow \infty}\left\|Q_{-}(-1) x_{n}(-1)\right\|=0 .
$$

Отсюда вытекает (см. формулу (8) и следствие 10), что

$$
\lim _{n \rightarrow \infty}\left\|\mathscr{A} x_{n}\right\|=0
$$

поэтому

$$
\lim _{n \rightarrow \infty}\left\|x_{n}\right\|=0
$$

Получено противоречие.

Пусть теперь равномерно инъективен оператор $\mathscr{D}$, но таким не является оператор $\Phi$. Это влечет существование нормированной последовательности векторов $\left(a_{n}\right)$ из $X_{-}^{\prime}(1)$ таких, что

$$
\lim _{n \rightarrow \infty}\left\|\Phi a_{n}\right\|=0 \text {. }
$$

Тогда последовательность $\left(x_{n}\right)$ из $\mathscr{F}(\mathbb{Z}, X)$ вида

$$
x_{n}(k)= \begin{cases}\mathscr{U}(k,-1) a_{n}, & k \leqslant-1, \\ \mathscr{U}(k, 0) P_{+}(0) U(0) a_{n}, & k \geqslant 0,\end{cases}
$$

обладает свойством $\inf _{n \geqslant 1}\left\|x_{n}\right\|>0$. Соотношения

$$
\left(\mathscr{D} x_{n}\right)(k)=0, \quad k \neq 0, \quad\left(\mathscr{D} x_{n}\right)(0)=-\Phi a_{n} \rightarrow 0, \quad n \rightarrow \infty,
$$

ведут к противоречию. Теорема доказана. 
СлЕДСТВИЕ 13. Если подпространство $X_{-}^{\prime}(-1)$ конечномерно (например, если компактен оператор $U(0)) u \operatorname{Ker} \Phi=\{0\}$, то оператор $\mathscr{D}$ равномерно инбективен.

СлеДСтвИЕ 14. Если подпространство $X_{0}=\operatorname{Ker} \Phi$ дополняемо в $X, m o \operatorname{Im} \mathscr{D}$ замкнутое подпространство из $\mathscr{F}(\mathbb{Z}, X)$, а подпространство $\operatorname{Ker} \mathscr{D}$ дополняемо в $\mathscr{F}(\mathbb{Z}, X)$.

2. Доказательство теорем 1-3. Фредгольмовость оператора $\mathscr{D}$. Для дальнейшего изучения свойств оператора $\mathscr{D} \in$ End $\mathscr{F}(\mathbb{Z}, X)$, связанных с его обратимостью, привлечем его сопряженный оператор $\mathscr{D}^{*} \in$ End $\mathscr{F}(\mathbb{Z}, X)^{*}$. При этом отметим, что банахово пространство $\ell_{p}(\mathscr{N}, X)^{*}, p \in[1, \infty)$, будет отождествляться (изоморфно) с пространством $\ell_{q}\left(\mathscr{N}, X^{*}\right)$, где $q=p(p-1)^{-1}, c_{0}(\mathscr{N}, X)^{*}-$ с пространством $\ell_{1}\left(\mathscr{N}, X^{*}\right)$. Если $\mathscr{D} \in \operatorname{End} \ell_{\infty}(\mathbb{Z}, X)$, то при доказательстве утверждений будет вначале рассматриваться его сужение на $c_{0}$.

Оператор $\mathscr{D}^{*}$ определяется равенствами

$$
\left(\mathscr{D}^{*} \xi\right)(n)=\xi(n)-U(n)^{*} \xi(n+1), \quad n \in \mathbb{Z}, \quad \xi \in \mathscr{F}^{*} .
$$

В следующей лемме используются операторы $\mathscr{B}_{r}, \mathscr{B}_{l}$, введенные в леммах 2,3 .

Лемма 4. Операторы $\overline{\mathscr{A}}=I-\mathscr{D} \mathscr{B} \in$ End $\mathscr{F}(\mathbb{Z}, X), \overline{\mathscr{A}}^{*}=I-\mathscr{B}^{*} \mathscr{D}^{*} \in$ End $\mathscr{F} *$ определяются следующими равенствами:

$$
\begin{gathered}
((I-\mathscr{D} \mathscr{B}) x)(0)=\sum_{m=0}^{\infty} \mathscr{U}(0, m) Q_{+}(m) x(m)+Q_{+}(0) U(0) \sum_{m=-\infty}^{-1} \mathscr{U}(-1, m) P_{-}(m) x(m), \\
((I-\mathscr{D} \mathscr{B}) x)(n)=0, \quad n \neq 0, \quad x \in \mathscr{F}(\mathbb{Z}, X), \\
\left(\overline{\mathscr{A}}^{*} \xi\right)(n)= \begin{cases}\mathscr{U}(0, n)^{*} Q_{+}(0)^{*} \xi(0), & n \geqslant 0, \\
\mathscr{U}(-1, n)^{*} P_{-}(-1)^{*} U(0)^{*} Q_{+}(0)^{*} \xi(0), & n \leqslant-1,\end{cases}
\end{gathered}
$$

əде $\xi \in \mathscr{F}^{*}$.

ДокАЗАтЕльСтво. Равенства, определяющие оператор $\overline{\mathscr{A}}=I-\mathscr{D} \mathscr{B}$, непосредственно следуют из определения операторов $\mathscr{D}$ и $\mathscr{B}$.

СледСтвиЕ 15. Оператор $\overline{\mathscr{A}}$ компактен, если конечномерен оператор $Q_{+}(0)$ (в частности, если компактен оператор $U(0)$ ).

Более подробно остановимся на рассмотрении операторов $\mathscr{D}^{*}$ и $\overline{\mathscr{A}}^{*}$. Отметим, что сопряженные пространства к подпространствам $X_{-}(n), X_{-}^{\prime}(n), n<0, X_{+}(n), X_{+}^{\prime}(n)$, $n \geqslant 0$, далее естественньм образом будут отождествляться с образами соответствующих проекторов $P_{ \pm}(n)^{*}, Q_{ \pm}(n)^{*}$.

Теорема 6. Если выполнено предположение 1, то ядро $\operatorname{Ker} \mathscr{D}^{*}$ оператора $\mathscr{D}^{*}$ состоит из последовательностей $\xi \in \mathscr{F}^{*}$ вида

$$
\xi(n)= \begin{cases}\mathscr{U}(0, n)^{*} \xi_{0}, & n \geqslant 0, \\ \mathscr{U}(-1, n)^{*} U(0)^{*} \xi_{0}, & n \leqslant-1,\end{cases}
$$

əде $\xi_{0} \in \operatorname{Ker} \Phi^{*} u \Phi^{*}: \operatorname{Im} Q_{+}(0)^{*} \rightarrow \operatorname{Im} Q_{-}(-1)^{*}-$ сопря женный оператор $к$ узловому оператору $\Phi: X_{-}^{\prime}(-1) \rightarrow X_{+}^{\prime}(0)$. 
ДоказАтЕльство. Ясно, что $\operatorname{Ker} \mathscr{D}^{*} \subset \operatorname{Ker} \mathscr{D}^{*} \overline{\mathscr{A}}^{*}$. Поскольку $\left(\mathscr{D}^{*} \overline{\mathscr{A}}^{*} \xi\right)(n)=0$ для всех $n \neq-1, \xi \in \mathscr{F}^{*}$, то

$$
\xi \in \operatorname{Ker} \mathscr{D}^{*} \Longrightarrow\left(\mathscr{D}^{*} \overline{\mathscr{A}}^{*} \xi\right)(-1)=0 \text {. }
$$

Поэтому из равенства

$$
\left(\mathscr{D}^{*} \overline{\mathscr{A}}^{*} \xi\right)(-1)=Q_{-}(-1)^{*} U(0)^{*} Q_{+}(0)^{*} \xi(0)
$$

следует, что $\xi \in \operatorname{Ker} \mathscr{D}^{*}$ в точности тогда, когда вьполняется равенство

$$
Q_{-}(-1)^{*} U(0)^{*} Q_{+}(0)^{*} \xi(0)=\Phi^{*} Q_{+}(0)^{*} \xi(0)=0
$$

т.е. когда $Q_{+}(0)^{*} \xi(0) \in \operatorname{Ker} \Phi^{*}$. Отсюда следует указанное в условии теоремы представление последовательностей из $\mathrm{Ker} \mathscr{D}^{*}$. Теорема доказана.

СЛЕДСТВИЕ 16. Имеет место равенство $\operatorname{dim} \operatorname{Ker} \mathscr{D}^{*}=\operatorname{dim} \operatorname{Ker} \Phi^{*}$.

Отметим, что верны соответствуюшие аналоги всех следствий теоремы 4.

ТЕОрема 7. Если выполнено предположение 1, то оператор $\mathscr{D}^{*}$ равномерно инбективен тогда и только тогда, когда равномерно инбективен оператор $\Phi^{*}$.

ДокАЗАТЕЛЬСТВО этой теоремы почти дословно повторяет доказательство теоремы 5.

Теперь приступим к непосредственному доказательству теорем 1-3.

ДоКАЗАТЕЛЬСТвО ТЕОРЕМЫ 1. Пусть $\mathscr{D}$ - обратимьй оператор из End $\mathscr{F}(\mathbb{Z}, X)$. Тогда из результатов статей [4], [5] следует, что $\mathscr{D}$ - обратимьй оператор из алгебры End $\ell_{\infty}(\mathbb{Z}, X)$. Поэтому в силу леммы 1 семейство допускает экспоненциальную дихотомию на $\mathbb{Z}$ и, следовательно, выполнены условия предположений 1 и 2 . Из обратимости оператора $\mathscr{D}$ следует обратимость оператора $\mathscr{D}^{*}$. Поэтому они оба равномерно инъективны и, следовательно, (в силу теорем 5 и 7) равномерно инъективны операторы $\Phi$ и $\Phi^{*}$. Отсюда следует (см. [6, § VI.6, теорема 2]) обратимость узлового оператора $\Phi$.

Пусть теперь вьполнены условия теоремы 1 . Тогда из теорем 5 и 7 следует (см. также теоремы 4 и 6), что $\mathscr{D}$ - равномерно инъективньй оператор и $\operatorname{Ker} \mathscr{D}^{*}=\{0\}$. Следовательно, еще раз используя теорему 2 из [6, §VI.6], получаем, что $\mathscr{D}$ - обратимый оператор. Теорема доказана.

Выполнение условий любого из следствий 1, 2 влечет обратимость оператора $\Phi$; поэтому $\mathscr{D}$ - обратимьй оператор. Если выполнено условие 2) следствия 2 , то имеет место разложение (2), причем проекторами, осуществляющими разложение $X=X_{-}^{-}(0) \oplus$ $X_{+}(0)$, являются проекторы

$$
\mathscr{P}_{-}=Q_{-}(0)\left(Q_{-}(0)-P_{+}(0)\right)^{-1}, \quad \mathscr{P}_{+}=-P_{+}(0)\left(Q_{-}(0)-P_{+}(0)\right)^{-1} .
$$

Таким образом, доказаны следствия 1 и 2. 
ДокАЗАТЕЛЬСТВО ТЕОРЕМЫ 2. Выполнение любого из условий теоремы ведет к выполнению условий теоремы 1 , а также к вьполнению условий любого из ее следствий 1 и 2. Отметим, что при выполнении условия 1$)$ теоремы следует положить $Q_{-}(0)=0$. Теорема доказана.

ЗАмЕчАниЕ 3 . Наличие тривиальной экспоненциальной дихотомии семейства $\mathscr{U}$ на множествах $\mathbb{Z}_{-}$и $\mathbb{Z}_{+}$со свойством $P_{-}=0, P_{+}=0$ (аналога свойства 1 ) теоремы 2) не гарантирует обратимости оператора $\mathscr{D}$. Примером может служить оператор $\mathscr{D} \in \operatorname{End} \ell_{p}(\mathbb{Z}, X)$ вида

$$
(\mathscr{D} x)(n)= \begin{cases}x(n)-2 x(n-1), & n \leqslant-1 \\ x(n)+2 x(n-1), & n \geqslant 1 \\ x(0), & n=0 .\end{cases}
$$

В матрице этого оператора, используемой при доказательстве теоремы 4 , оператор $\mathscr{D}_{0}$ нулевой. Поэтому $\sigma(\mathscr{D})=\{1+\lambda|| \lambda \mid \leqslant 2\}$. Следовательно, $\mathscr{D}$-необратимьй оператор.

ДокАЗАТЕЛЬСтво тЕоремы 3. Докажем, что вьполнено условие 1) теоремы 2 , т.е. для семейства $\mathscr{U}$ имеет место тривиальная дихотомия на $\mathbb{Z}$ и $_{\mathbb{Z}_{+}}$с $Q_{-}=0$ и $Q_{+}=0$. Поскольку $r_{0}=\max \left\{r\left(U_{ \pm}\right), r\left(U_{-}\right)\right\}<1$, для любого $r \in\left(r_{0}, 1\right)$ сушествует постоянная $C(r)>0$ такая, что имеют место оценки (см. [6, гл. VII])

$$
\left\|U_{ \pm}^{n}\right\| \leqslant C(r) r^{n}, \quad n \geqslant 1
$$

Пусть $B_{n}=U(n)-U_{+}, n \geqslant 1$. Тогда $\varepsilon_{n}=\left\|U(n)-U_{+}\right\| \rightarrow 0$ при $n \rightarrow \infty$, поэтому для числа $\varepsilon>0$, удовлетворяющего условию $q=r+\varepsilon<1$, существует $n_{0} \in \mathbb{N}$ такое, что $\varepsilon_{n} \leqslant \varepsilon$ при всех $n \geqslant n_{0}$. Для $n \geqslant m \geqslant n_{0}$ имеют место оценки

$$
\|\mathscr{U}(n, m)\|=\left\|\left(U_{+}+B_{n}\right) \cdots\left(U_{+}+B_{m+1}\right)\right\| \leqslant C(r)(r+\varepsilon)^{n-m-1}=C_{1}(r) q^{n-m},
$$

где $C_{1}(r)=C(r) q^{-1}$.

Теперь для произвольных $n \geqslant m$ из $\mathbb{Z}_{+}$получаем оценку $\|\mathscr{U}(n, m)\| \leqslant M q^{n-m}$, где

$$
M=C(r)\left(\prod_{k=0}^{n_{0}}\|U(k)\|\right) q^{-n_{0}} .
$$

Таким образом, семейство эволюционных операторов $\mathscr{U}$ допускает тривиальную экспоненциальную дихотомию на множестве $\mathbb{Z}_{+}$c $Q_{+}=0$. Аналогично доказьвается наличие тривиальной экспоненциальной дихотомии у семейства $\mathscr{U}$ на множестве $\mathbb{Z}_{-}$ с $Q_{-}=0$. Теорема доказана.

ЗАмЕчАниЕ 4. Если $\mathscr{F}(\mathbb{Z}, X)=\ell_{\infty}(\mathbb{Z}, X)$, то все утверждения, связанные с использованием сопряженного оператора $\mathscr{D}^{*}$, доказываются с помощью рассмотрения сужения оператора $\mathscr{D}$ на подпространство $c_{0}$ (при этом сохраняются его ядро, свойство равномерной инъективности и т.д.). Если доказана обратимость оператора $\mathscr{D}$ на $c_{0}$, то он обратим и на $\ell_{\infty}($ см. [4], [5]). То же верно и для большинства рассматриваемых свойств оператора $\mathscr{D}$. 
ТЕОрема 8. Пусть выполнено предположение 1. Тогда оператор Д фредгольмов (полуфредгольмов) тогда и только тогда, когда фредгольмовым (полуфредгольмовым) является оператор $\Phi$. Если $\Phi$ фредгольмов, то индексы операторов $\Phi$ u $\mathscr{D}$ совпадают.

ДокАЗАТЕЛЬСТвО утверждений теоремы непосредственно вытекает из следствия 6 , теоремы 4 и ее следствия 14, из теоремы 6 и ее следствия 16, а также из теоремы 7.

СлЕДСТВИЕ 17. Индекс фредгольмова оператора $\mathscr{D}$ не зависит от выбора пространства $\mathscr{F}(\mathbb{Z}, X)$.

3. О некоторых обобщениях полученных результатов. Будем говорить, что семейство $\mathscr{U}$ допускает әкспоненииальную дихотомию в точке $\infty$, если существует $m \in \mathbb{Z}$ такое, что $\mathscr{U}$ допускает экспоненциальную дихотомию на множестве $\mathbb{Z}+(m)=$ $\{m, m+1, m+2, \ldots\}$ (в этом случае расщепляющая пара проекторнозначных функций обозначается $\left.\left(P_{+}, Q_{+}\right)\right)$. Аналогичное определение дается для точки $-\infty$, и соответствующая расщепляющая пара обозначается $\left(P_{-}, Q_{-}\right)$.

Отметим несколько случаев, когда имеет место экспоненциальная дихотомия в точках $-\infty$ и $+\infty$.

1) Оператор $\mathscr{D} ф$ редгольмов и $X$ - конечномерное пространство (в этом случае обратимы все операторы из множества всех предельных операторов [7], построенных по $\mathscr{D}$, что влечет экспоненциальную дихотомию для соответствующих семейств эволюционных операторов и, следовательно, экспоненциальную дихотомию в точках $\pm \infty)$.

2) Сушествуют числа $m_{1}<0$ и $m_{2}>0$ из $\mathbb{Z}$ такие, что

$$
\sup _{n \geqslant m_{2}}\left\|\mathscr{U}\left(n, n-m_{2}\right)\right\|<1, \quad \sup _{n \leqslant 0}\left\|\mathscr{U}\left(n, n+m_{1}\right)\right\|<1
$$

(выполнение этих неравенств означает наличие тривиальной экспоненциальной дихотомии в точках $\pm \infty$ с $Q_{+}=0, Q_{-}=0$; см. [2, теоремы 2 и 6]).

3) Существуют (в равномерной операторной топологии) пределы

$$
\lim _{n \rightarrow \pm \infty} U(n)=U_{ \pm} \in \text { End } X
$$

и $\sigma\left(U_{ \pm}\right) \cap \mathbb{T}=\oslash$, где $\mathbb{T}=\{\lambda \in \mathbb{C}|| \lambda \mid=1\}$.

4) Оператор $\mathscr{D}_{-} \in$ End $\mathscr{F}\left(\mathbb{Z}_{-}, X\right)$ сюръективен, оператор $\mathscr{D}_{+} \in$ End $\mathscr{F}\left(\mathbb{Z}_{+}, X\right)$ равномерно инъективен и подпространства из $X$ вида

$$
X_{-}(-1)=\left\{x(-1) \mid x \in \operatorname{Ker} \mathscr{D}_{-}\right\}, \quad X_{+}(0)=\left\{x_{0} \in X \mid \sup _{n \geqslant 1}\left\|\mathscr{U}(n, 0) x_{0}\right\|<\infty\right\}
$$

дополняемы в $X$ (в этом случае для семейства $\mathscr{U}$ имеет место экспоненциальная дихотомия на множествах $\mathbb{Z}_{-}$и $\mathbb{Z}_{+}$; см., например, [2, теорема 6$\left.]\right)$.

Пусть семейство $\mathscr{U}$ допускает экспоненциальную дихотомию в точках $-\infty$ и $\infty$ с определяющими ее числами $m_{1}$ и $m_{2}$ из $\mathbb{Z}$, для которых $m_{1}<m_{2}$. Зададим оператор $\Phi\left(m_{1}, m_{2}\right): X_{-}^{\prime}\left(m_{1}\right) \rightarrow X_{+}^{\prime}\left(m_{2}\right)$ формулой

$$
\Phi\left(m_{1}, m_{2}\right) x=Q_{+}\left(m_{2}\right) \mathscr{U}\left(m_{2}, m_{1}\right) Q_{-}\left(m_{1}\right) x, \quad x \in X_{-}^{\prime}\left(m_{1}\right) .
$$


Все полученные в условиях предположения 1 результаты статьи могут быть сформулированы в терминах соответствующих свойств оператора $\Phi\left(m_{1}, m_{2}\right)$. Так, например, оператор $\mathscr{D}$ обратим тогда и только тогда, когда обратим оператор $\Phi\left(m_{1}, m_{2}\right)$. Сохраняется и формула индекса для фредгольмова оператора $\mathscr{D}$ (т.е. ind $\mathscr{D}=$ ind $\Phi\left(m_{1}, m_{2}\right)$ ).

В заключение отметим, что полученные в данной статье результаты могут найти применение в исследовании обратимости и фредгольмовости абстрактных параболических операторов (см., например, [8, теорема 3]).

\section{СПИСОК ЦИТИРОВАННОЙ ЛИТЕРАТУРЫ}

[1] Хенри Д. Геометрическая теория полулинейных параболических уравнений. М.: Мир, 1985.

[2] Баскаков А.Г. О корректности линейных дифференциальных операторов // Матем. сб. 1999. T. 190. № 3. C. 3-28.

[3] ЖКиков В. В. Некоторые вопросы допустимости и дихотомии. Принцип усреднения // Изв. АН СССР. Сер. матем. 1976. Т. 40. №6. С. 1380-1408.

[4] Баскаков А.Г. Абстрактный гармонический анализ и асимптотические оценки элементов обратных матриц // Матем. заметки. 1992. Т. 52. № 2. С. 17-25.

[5] Курбатов В.Г. Об алгебрах разностньх и интегральных операторов // Функцион. анализ и его прилож. 1990. Т. 24. № 2. С. 98-99.

[6] Данфорд Н., Шварц Дж. Т. Линейные операторы. Т. І. М.: ИЛ, 1962.

[7] Рабинович В. С. Операторные дискретные свертки и некоторые их приложения // Матем. заметки. 1992. Т. 51. №1. С. 90-101.

[8] Баскаков А. Г. Полугруппы разностных операторов в спектральном анализе линейных диффференциальных операторов // Функцион. анализ и его прилож. 1996. Т. 30. №3. С. 1-11.

Воронежский государственный университет 